\title{
Cascading failure spreading on weighted heterogeneous networks
}

\author{
Zhi-Xi Wu, Gang Peng, Wen-Xu Wang, Sammy Chan, and Eric \\ Wing-Ming Wong $\ddagger$ \\ Department of Electronic Engineering, City University of Hong Kong, Hong Kong SAR, \\ People's Republic of China
}

\begin{abstract}
We study the onset and spreading of cascading failure on weighted heterogeneous networks by adopting a local weighted flow redistribution rule, where the weight and tolerance of a node is correlated with its link degree $k$ as $k^{\theta}$ and $C k^{\theta}$, respectively. The weight parameter $\theta$ and tolerance parameter $C$ are positive: $\theta>0$ and $C>1.0$. Assume that a failed node leads only to a redistribution of flow passing through it to its nearest neighboring nodes. We give out theoretical estimations of the onset of the cascading failure for different values of $\theta$. It is found that the cascading failure emerges most difficult on networks with $\theta=1.0$, while it develops more slowly for larger $\theta$. We furthermore explore the statistical characteristics of the avalanche size on the networks by varying $\theta$, and obtain versatile dynamical scenarios of the cascading processes, which exhibit either "subcritical", or "critical", or "supercritical" behaviors depending on the value of the weight parameter.
\end{abstract}

PACS numbers: $89.75 . \mathrm{Hc}$

Submitted to: J. Stat. Mech.

‡ Corresponding author: eeewong@ cityu.edu.hk 


\section{Introduction}

Network science focusing on the relationship between structure, function and dynamics has attracted much attention in recent years $[1,2,3,4]$. One of the hot topics in the network science is the robustness characteristic of complex network against attacks and random failures $[1,2,3,4]$, as known that our daily life are closely related to various types of networks, such as power grids, information communication networks, and transportation networks, and so on. Evidence has demonstrated that in such networks, even though intentional attacks or random failures emerge very locally, the entire network can be greatly affected, often resulting in large scale collapse and unfunctioning of part or whole of the systems. An often cited realistic example is the 1996 blackout of the power transportation network in U.S.A. [5].

Up to now, it has been shown again and again that the topological features of underlying interaction networks have great impacts on the final outcomes of the dynamics taking place on them $[1,2,3,4]$. For example, the scale free topology of a network results in a vanishing threshold of epidemic spreading on it with the increase of the network size, also gives rise to a robust behavior against random failures and fragile for aimed attacks $[1,3]$. In fact, understanding how the structure affects the dynamics is regarded as one of the major objectives in the study of complex networks [2]. For this reason, most previous cascading models confined on complex networks, such as the sandpile model [6], the global load based cascading model $[7,8,9,10,11,12,13]$, and the fiber bundle model $[14,15,16]$, have also been subjected to this issue. However, the network weights have not been taken into consideration in these models, regardless of the fact that real networks display a large heterogeneity in the weights which have a strong correlation with the network topology $[17,18,19]$. In fact, the existing common weighted features play a significant role in a variety of dynamical processes, including epidemic spreading, information packets routing, synchronization, etc [20, 21, 22, 23]. In particular, Wang et al. [21] introduced a localinformation based routing strategy on scale free networks, wherein each node is selected as a router by its neighbors with a probability proportional to its weight $k^{\alpha}$. They showed that in the case of $\alpha=-1.0$, the whole network achieves its optimal performance. Another influential work concerning about alternative routing strategy on networks is studied by Yan et al. [22], in which each node contributes a weight of $k^{\beta}$ to any path going through it. It was found at $\beta=1.0$ the network capability in processing traffic is improved more than 10 times as compared to shortest path routing. More recently, Korniss [23] proposed that complex networks are easy to be synchronized if the coupling strength (denoted by the weight of the edges) between any two connected nodes with degrees $k_{i}$ and $k_{j}$ is weighted as $\left(k_{i} k_{j}\right)^{-1.0}$. Hence, there is a need for a modelling approach that can capture the coupling of cascading and weighted characteristics.

In a recent work of Wang and Chen [24], a cascading model with a local weighted flow redistribution rule (LWFRR) is proposed and studied on weighted scale free and small world networks. In their model, the cascading process is triggered by a small initial perturbation (a randomly selected edge with weight $\left(k_{i} k_{j}\right)^{\theta}$ is attacked), and spreads to other constituents sequentially. They found that the weighted complex network reaches 
the strongest robustness level at a universal value of the weight parameter $\theta=1$. In the present work, we follow the research of [24] by considering a cascading model on weighted complex heterogeneous networks, wherein the cascading process is triggered by small initial attacks or failures on network nodes. It is found that in the node-based cascading model, the condition for the universal robustness of the network is unchanged. In addition, by exploring the statistical characteristics of the avalanche size, we obtain versatile scenarios of the cascading dynamical processes, which exhibit either "subcritical", "critical", or "supercritical" behaviors depending on the value of the weight parameter.

\section{The model}

It has been proposed that many realistic networks share two common properties: small average path lengths among any two nodes and a power-law degree distribution [1, 2, 3, 4]. For simplicity, we use the well-known Barabási-Albert (BA) scale-free network model [1] as the physical infrastructure on top of which a cascading process takes place. The BA model containing two generic mechanisms of many real complex systems: growth and preferential attachment [1] can be constructed as follows. Starting from $m_{0}$ nodes, one node with $m$ links is attached at each time step in such a way that the probability $\prod_{i}$ of being connected to the existing node $i$ is proportional to the degree $k_{i}$ of that node, i.e., $\prod_{i}=k_{i} / \sum_{j} k_{j}$, where $j$ runs over all existing nodes. In the present work, the total network size is fixed as $N=5000$ and the parameters are set to be $m_{0}=m=2$ (hence the average connectivity of the network is $\langle k\rangle=2 m=4[1])$.

With the heterogeneous networks at hand, let us define the cascading model under the LWFRR based on node failure. We assume that the weight of a node $i$ is given by $w_{i}=k_{i}^{\theta}$, where $\theta$ is a tunable parameter in our study, which controls the strength of the node weight. This assumption is reasonable since many previous studies concerning about both model networks and real networks have shown that the load of (or traffic handled by) a node scales with its degree as $L(k)=b k^{\eta}$, where $\eta$ relies on topological elements [11, 17, 25, 26, 27]. Thus, our assumption on the node weights is in accordance with the previous load based model but has practical convenience. Following Ref. [24], we assume that the potential cascading failure is triggered by a small initial attack or perturbation, e.g., unfunctioning of a single node $i$. The flow supposed to going through the broken node $i$ will be redistributed to its nearest neighboring nodes (see Fig. 1 for illustration). The additional flow $\Delta F_{j}$ received by the neighboring node $j$ is proportional to its weight,

$$
\Delta F_{j}=F_{i} \times \frac{F_{j}}{\Sigma_{l \in \Omega_{i}} F_{l}},
$$

where $\Omega_{i}$ is the set of neighboring nodes of $i$. Following previous models addressing cascading problems $[8,10,13,16,24]$, each node $i$ in the network has a weight threshold or capacity $\mathcal{F}_{i}$, which is the maximum flow that the node can handle. Conventionally, it is assumed that the threshold of a node is proportional to its weight $\mathcal{F}_{i}=C F_{i}=C k_{i}^{\theta}$, where the constant $C>1$ is a threshold parameter characterizing the tolerance of the network 
$[7,8,9,10,13,16,24]$. Considering a neighbor $j$ of the node $i$, if

$$
F_{j}+\Delta F_{j}>\mathcal{F}_{j}=C k_{j}^{\theta}
$$

then the node $j$ will be broken, inducing further redistribution of flow of $F_{j}+\Delta F_{j}$ and potentially further nodes' breakdown. After the cascading failure process stops, we calculate the avalanche size $S_{i}$, which is defined as the total number of broken nodes through the process induced by attacking the node $i$ initially.

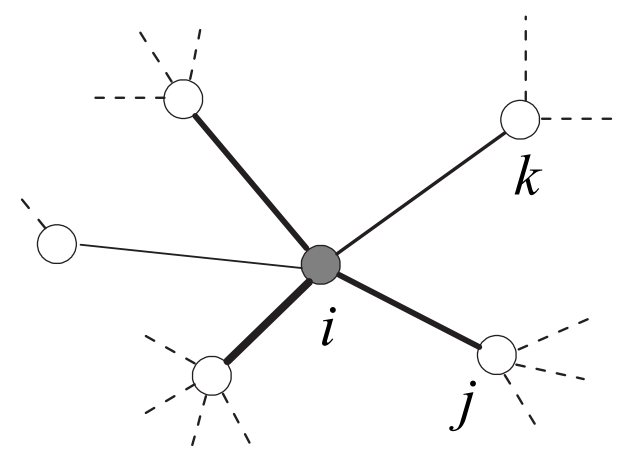

Figure 1. Schematic illustration of the LWFRR triggered by a node failure. The focal node $i$ is broken and the flow along it is redistributed to its neighboring nodes $j, k, \ldots$. Among these neighbors, the one with higher flow capacity (i.e., more degrees) will undertake more shared flow (denoted by the width of the links) from the failed node.

The LWFRR can be explained by taking the scenario of information traffic on the Internet as an example. After congestion or breakdown occurs in a router, information flow is rerouted to bypass it, which evidently leads to the flow increase in other routers. Since, in general, a node of higher traffic flows has always a stronger ability to handle traffic transmission, i.e., a node's threshold is proportional to its weight, it is reasonable to preferentially reroute traffic along those higher-capacity nodes to maintain normal functioning of traffic and try to avoid further congestions. For simplicity, we assume that the additional flow received by a router is proportional to its weight. When a node receives extra flow, its total flow may exceed its capacity with packets built up in its buffer, and congestion occurs consequently. The same story could happen again for those neighbors of the newly broken nodes. As a result, an avalanche of overloads emerges on the network. Another related example for LWFRR exists in our daily public traffic system. If we map each road as a node, and the intersection between two roads as a link between them, then we can get a traffic network [28]. Once a road is congested because of some traffic accidents, the congestion of the road will immediately increase the burden of adjacent roads. It is natural that those roads with more traffic lanes would share more additional traffic. At last, we want to remark that our LWFRR may be more relevant to the dynamics in the protein interaction network or cellular networks. In such systems, when a certain element such as a protein or a substrate fails or is removed, others should take over its burden to survive the lack thereof. 


\section{Results and discussion}

To explore the effect of a small initial attack on our cascading model, we defunctionalize only one node initially and calculate $S_{i}$, which is the avalanche size (the total number of broken nodes) induced by defunctionalizing $i$, after the cascading process is over. To quantify the robustness of the network, we adopt the average avalanche size $S=\sum_{i} S_{i} / N$, obtained via summation over all the avalanche sizes by defunctionalize each node initially at each time divided by the total number of nodes $N$. Given a value of $\theta$, when the value of $C$ is sufficiently small, we can imagine that it is easy for the whole network to get into fully collapse in the case of arbitrary node's failure. On the other hand, for sufficiently large $C$, the destructive impacts caused by the failure of those nodes with small degrees could be absorbed by nodes with large ones, and no cascading phenomenon emerges. Thus, with the increase of $C$, there should be some crossover behavior of the system from large scale breakdowns to no breakdown, going through small scale ones. Thus, $S$ can be regarded as an order parameter to characterize the robustness of the network.

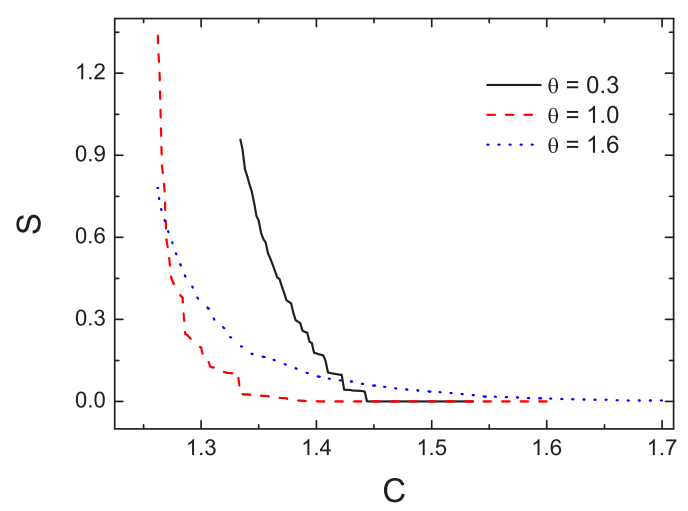

Figure 2. Average avalanche size $S$ as a function of tolerance parameter $C$ for serval values of $\theta$ on BA scale free networks with total size $N=5000$ and average connectivity $\langle k\rangle=4$. The cascading failure threshold $\mathcal{C}$ beyond which broken nodes' growth occurs $(S>0)$ is smaller for $\theta=1.0$ as compared to the cases of $\theta=0.3$ and 1.6. Each curve is obtained by averaging over experiments on 20 independent networks.

Figure 2 shows $S$ as a function of the tolerance parameter $C$ for serval values of $\theta$ for BA networks with size $N=5000$ and average connectivity 4 . Each curve is obtained by averaging over experiments on 20 independent networks. For each curve, a crossover behavior occurs at a critical threshold of $C$. When the value of $C$ is beyond this threshold, no cascading failure arises and the system maintains its normal and efficient functioning; While for the case of $C$ smaller than the threshold, $S$ increases rapidly from zero and cascading failure emerges, causing the whole or part of the network to stop working. Hence the threshold is the least value of protection strength to avoid cascading failure. Apparently, the lower the value of it, the stronger the robustness of the network against cascading failure. 
From Figure 2, we note that it is the case of $\theta=1.0$ that a cascading process occurs at the latest with the decrease of $C$, which is in accordance with the edge-based cascading model [24]. In order to understand this observed universal phenomenon, we provide some theoretical analysis. To avoid the emergence of cascading failure, the following condition should be satisfied:

$$
\frac{F_{i} F_{j}}{\sum_{l \in \Omega_{i}} F_{l}}+F_{j}<\mathcal{F}_{j}
$$

Rewriting the arguments as a function of the node degree, we have

$$
\frac{k_{i}^{\theta} k_{j}^{\theta}}{\sum_{l \in \Omega_{i}} k_{l}^{\theta}}+k_{j}^{\theta}<C k_{j}^{\theta} \text {. }
$$

The denominator of the first term of the above inequality can be written as

$$
\sum_{l \in \Omega_{i}} k_{l}^{\theta}=\sum_{k^{\prime}=k_{\min }}^{k_{\max }} k_{i} P\left(k^{\prime} \mid k_{i}\right) k^{\prime \theta},
$$

where $P\left(k^{\prime} \mid k_{i}\right)$ is the conditional probability that a node of $k_{i}$ has a neighbor of $k^{\prime}$. Since BA networks have no degree-degree correlation [2], we have $P\left(k^{\prime} \mid k_{i}\right)=k^{\prime} P\left(k^{\prime}\right) /\langle k\rangle$. Combining (4) and (5), we yield

$$
\frac{k_{i}^{\theta-1}\langle k\rangle}{\left\langle k^{\theta+1}\right\rangle}+1<C
$$

From the above inequality, the critical tolerance parameter, denoted by $\mathcal{C}$, can be calculated by considering the ranges of $\theta<1, \theta=1$, and $\theta>1$, respectively:

$$
\mathcal{C}= \begin{cases}k_{\max }^{\theta-1}\langle k\rangle /\left\langle k^{\theta+1}\right\rangle+1, & \theta>1, \\ \langle k\rangle /\left\langle k^{2}\right\rangle+1, & \theta=1, \\ k_{\min }^{\theta-1}\langle k\rangle /\left\langle k^{\theta+1}\right\rangle+1, & \theta<1,\end{cases}
$$

where $k_{\max }$ and $k_{\text {min }}$ are the maximum and minimum node degrees of the network. To find the rank of these critical values, we first consider the ratio of $\mathcal{C}(\theta>1)-1$ to $\mathcal{C}(\theta=1)-1$ :

$$
\frac{\mathcal{C}(\theta>1)-1}{\mathcal{C}(\theta=1)-1}=\frac{k_{\text {max }}^{\theta-1}\left\langle k^{2}\right\rangle}{\left\langle k^{\theta+1}\right\rangle} .
$$

Since the degree distribution of BA networks in the large limit size $N$ is $P(k)=2 k_{\min }^{2} k^{-3}$ [1], we have $k_{\text {max }}=k_{\text {min }}^{2} \ln N,\left\langle k^{2}\right\rangle=\int_{k_{\min }}^{k_{\text {max }}} k^{2} P(k) d k=k_{\min }^{2} \ln N$, and $\left\langle k^{\theta+1}\right\rangle=$ $\int_{k_{\min }}^{k_{\max }} k^{\theta+1} P(k) d k=\frac{2}{\theta-1} k_{\min }^{\theta+1}\left(N^{\frac{\theta-1}{2}}-1\right)$. Substituting these expressions to Eq.(8), we obtain

$$
\frac{\mathcal{C}(\theta>1)-1}{\mathcal{C}(\theta=1)-1}=\frac{(\theta-1) N^{\frac{\theta-1}{2}} \ln N}{2\left(N^{\frac{\theta-1}{2}}-1\right)} .
$$

In the large limit of $N$, both the denominator and nominator approach infinity, Eq.(9) can be calculated by deviating the denominator and nominator with respect to $N$, respectively, which yields $1+\ln N^{\frac{\theta-1}{2}}$. For $\theta>1$, Eq.(9) is larger than 1, which indicates $\mathcal{C}(\theta>1)>\mathcal{C}(\theta=1)$. Similarly, we can get $\mathcal{C}(\theta<1)>\mathcal{C}(\theta=1)$. Thus, for $\theta=1$, the cascading failure occurs most difficult with the decrease of the tolerance parameter $C$, confirming our simulation results in Figure 2. Here, we want to point out that there is some discrepancy between the 


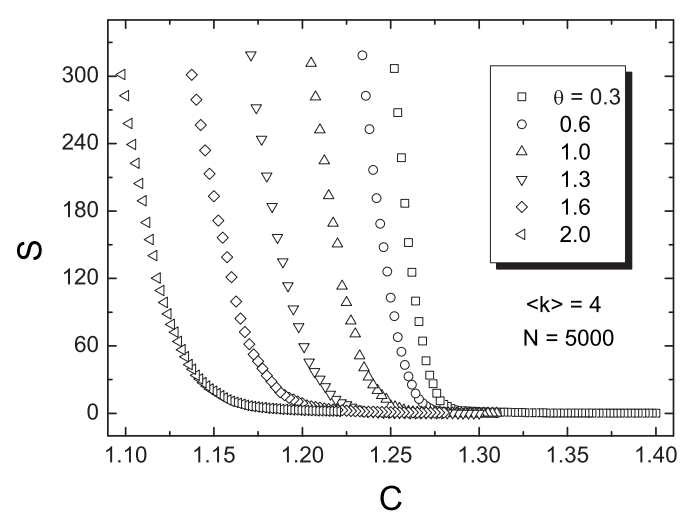

Figure 3. Average averge avalanche size $S$ as a function of the tolerance parameter $C$ on BA scale free networks with size $N=5000$. The average degree of the networks is fixed as $\langle k\rangle=4$, and the value of the weight parameter $\theta$ is varied as $0.3,0.6,1.0,1.3,1.6,2.0$, from right to left. The data are obtained by averaging over experiments on 20 independent networks

theoretical estimations of the critical tolerance parameter and those obtained by computer simulation, which results from one approximation in the analytical treatment and also the finite size of the networks.

So far, we have shown by both simulation and analysis that when the weight parameter $\theta=1.0$, cascading failure triggered by attacking a single node occurs most difficult on BA scale free networks. But it is not the whole story. Generally, besides the onset of the avalanche, we also care about how the avalanche size changes depending on the tolerance parameter. To this end, we also investigated the evolution of the average avalanche size on the network after the emergence of cascading failure by further decreasing $C$ while keeping the weight parameter $\theta$ fixed. The simulation results are presented in Figure 3. It is found that on networks with larger $\theta$ the average avalanche size develops in a slower rate as compared with those cases of smaller $\theta$. This point is reflected by the smaller avalanche size for larger value of $\theta$ at a given tolerance $C$. The phenomenon can be understood as follows. For heterogeneous networks with a large value of wight parameter $\theta$, if the nodes with large degrees are initially attacked, due to their high possessing capacity, it is easier for them to induce further breakdown of other nodes, especially those with smaller degrees. This gives rise to the result that cascading failure is easier to occur for large $\theta$, as was shown in Figure 2. On the other hand, for larger $\theta$, if it was nodes with smaller degrees that are attacked initially, the additional flow they released to their neighbors would be more easily absorbed by those neighbors with large degrees due to their higher tolerance, possibly without inducing further cascading failures. Moreover, it is known that in BA scale free networks, the nodes with large degrees only occupy a very small portion and most nodes are with small ones. Note that the average avalanche size $S$ is obtained by averaging over $S_{i}$ of all nodes in the network. Combining these elements, we can conclude that the networks with larger weight 
parameter is prone to cascading failure caused by single node's breakdown, whereas, after cascading failures emerged, their magnitudes develop more slowly as compared to the cases of smaller weight parameter. As for $\theta$ smaller than unity, since the capacity of high degree nodes are considerably inhibited, the high degree nodes are more easily broken induced by the failures of their small degree neighbors, i.e., the damage from small degree neighbors is more difficult to be absorbed by high degree nodes (for example, in the case of $\theta=0.1$, the absolute difference of the weight between a node with degree 4 and a node with degree 100 is about 0.436 , while for $\theta=1.0$ the difference is 96 ). Hence, the avalanche size grows more rapidly with decrease of $C$ than the case of $\theta=1$, as reflected in Figure 3.

Now let us investigate the avalanche size distribution $P(S)$, which is a conventional parameter to characterize the avalanche dynamics. To explore the statistical features of the avalanche sizes, we continuously increase the flow along nodes by a small constant $\delta$, starting from a load-empty network. This method is essentially the same with the sandpile model and fiber bundle model $[6,15,16]$. For simplicity, the threshold of each node is assigned to be its weight. At any time step, we add $\delta$ to the flow of one randomly selected node. With time going on, the flow accumulated on the network increases continuously. If the flow of a node exceeds its prescribed threshold, the node is broken and the flow on it will be redistributed to its neighboring nodes according to the LWFRR, which would induce other potential breakdown of the nodes. A sampling time step is counted after all the cascading events (if any) are over. At each time step, we record the number of broken nodes as the avalanche size at this time step. Then we recover all broken nodes and set their flows to zero. At the next time step, we add flow $\delta$ to a randomly selected nodes as before, and repeat the cascading process. The total sampling time is up to $10^{8}$. After that, we obtain the avalanche distribution of the sizes recorded at each time step. if no cascading failures occur, $S$ is zero.

We have explored the avalanche size distribution $P(S)$ on BA scale free networks with $N=5000$ and $\langle k\rangle=4$. The parameter $\theta$ is varied in the range $0.1 \leq \theta \leq 1.8$, while the parameter $\delta$ is fixed to $\delta=1$. The simulation results are shown in Figure 4. As can be seen from Figure 4, the form of the calculated $P(S)$ depends on the $\theta$-value considerably. For smaller $\theta, P(s)$ shows an exponential decay behavior bending down rapidly at larger magnitudes of $S$, which implies large avalanche events are scarce [Figure 4(a)]. Such behavior of $P(S)$ is often called "subcritical". For the moderate $\theta$, e.g., $\theta=0.9,1.0$, the curves lie on a straight line fairly well [Figure 4(b)], apparently satisfying the power law decay form, which is a prominent phenomenon empirically observed in previously studied cascading failure process and the mark of emergence of "criticality" [7, 9]. The truncation at the tails are due to finite size effect. As shown in Figure 4(c), for large values of $\theta, P(S)$ exhibits a pronounced peak structure for larger cascading events, while the power-law feature still remains for smaller magnitudes. Accordingly, such behavior of $P(S)$ is often called "supercritical", since $P(S)$ bends up at larger magnitudes (though it eventually falls off at even larger magnitudes), which means that a small initial perturbation can disturb a very large area of the whole system. Here, we want to remark that with the increase of $\theta$, both the power-law decay behavior of $P(S)$ and the pronounced peak where $P(S)$ bending up emerges smoothly, and the magnitude of the peak value also increases gradually [from Figure 

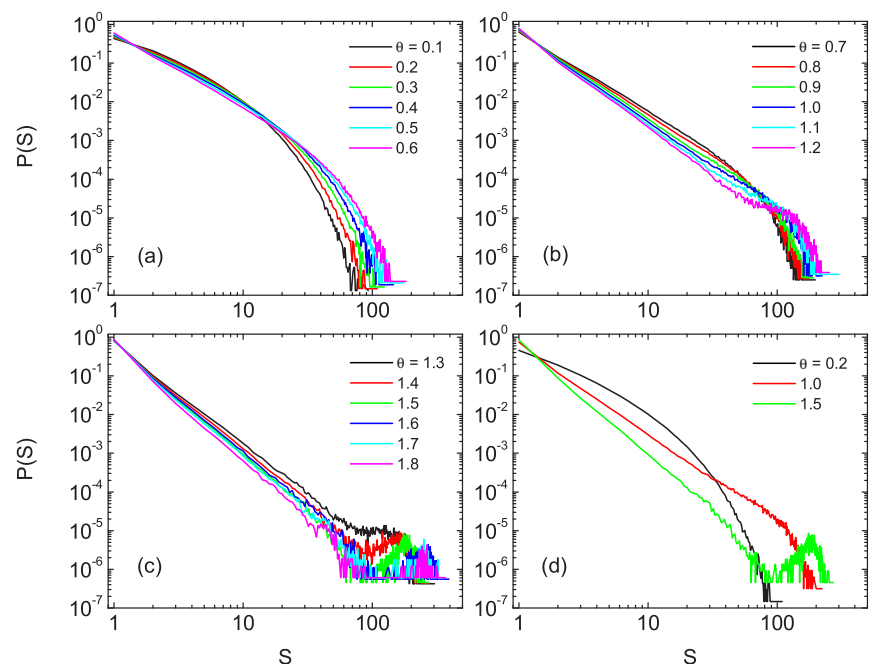

Figure 4. Distributions of the avalanche size from time step $t=1$ to $10^{8}$ for different values of $\theta$ on $\mathrm{BA}$ networks with size $N=5000$ and average degree $\langle k\rangle=4$. (a) represents $P(s)$ for smaller values of the weight parameter $0.1 \leq \theta \leq 0.6$; (b) represents $P(s)$ for moderate values of the weight parameter $0.7 \leq \theta \leq 1.2$; and (c) represents for larger values of the weight parameter $1.3 \leq \theta \leq 1.8$. (a), (b), and (c) correspond to the regions of "subcritical", "coexistence of subcritical-critical-supercritical", and "supercritical", respectively. For clarification, three typical curves in each region are shown in panel (d), corresponding to $\theta=0.2,1.0,1.5$, respectively.

4(a) to (c). For clarification, we show three typical curves in each region in Figure 4(d), corresponding to $\theta=0.2,1.0,1.5$, respectively]. Thus, the crossover from the "subcritical" to "critical" to "supercritical" behavior with increasing $\theta$ is of continuous or "second-order" nature. At present time, unfortunately, it is hard for us to give an analytical expression to $P(S)$, since the cascading process considered here is not a type of conventional branching process and cannot be addressed by following the method proposed in Ref. [25].

\section{Conclusion}

To sum up, we have studied the cascading reaction behaviors on BA scale free networks with respect to small node initial attacks. Each node is endowed with a weight $k^{\theta}$ describing its ability of handling load, and a tolerance $C k^{\theta}$ when confronting additional flow. We provide theoretical estimations of the critical tolerance parameter value for the onset of the cascading failure. It is found that the cascading failure emerges most difficult on networks with $\theta=1.0$. Though, with the decreasing of the tolerance, the networks with large value of $\theta$ are prone to the emergence of avalanche triggered by a randomly attacked node, the magnitude of avalanche size on them grows more slowly as compared to that on the networks with small $\theta$. Depending on the weight parameter $\theta$, subcritical, critical and supercritical avalanche 
dynamics may occur. For large $\theta$, cascading failure can spread over a large portion of the network. In other words, the smaller the value of $\theta$, the smaller region the cascading failure is confined. These results indicate the significant roles of weights in complex networks for designing protection strategies against cascading failures. In addition, the obtained powerlaw distributions of the avalanche sizes in critical and supercritical regimes demonstrate the validity and generality of our model for characterizing cascading reaction behaviors. These results may help us understand cascading phenomena in the real world, and may shed light on designing control strategy to prevent various cascading-failure-induced disasters.

\section{Acknowledgments}

We thank our colleague Prof. Kai-Hau Yeung for useful discussions.

\section{References}

[1] Albert R and Barabási A-L, 2002 Rev. Mod. Phys. 7447

[2] Newman M E J, 2003 SIAM Rev. 45167

[3] Boccaletti S, Latora V, Moreno Y, Chavez M and Hwang D-U, 2006 Phys. Rep. 424175

[4] Newman M E J, Barabási A-L and Watts D J, The Structure and Dynamics of Networks (Princeton University Press, Princeton, NJ, 2006).

[5] Sachtjen M L, Carreras B A and Lynch V E, 2000 Phys. Rev. E 614877

[6] Goh K-I, Lee D-S, Kahng B and Kim D, 2003 Phys. Rev. Lett. 91148701

[7] Motter A E and Lai Y C, 2002 Phys. Rev. E 66 065102(R)

[8] Motter A E, 2004 Phys. Rev. Lett. 93098701

[9] Lee E J, Goh K-I, Kahng B and Kim D, 2005 Phys. Rev. E 71056108

[10] Zhao L, Park K and Lai Y C, 2004 Phys. Rev. E 70 035101(R)

[11] Zhao L, Park K, Lai Y C and Ye N, 2005 Phys. Rev. E 72 025104(R)

[12] Crucitti R, Latora V and Marchiori M, 2004 Phys. Rev. E 69 045104(R)

[13] Schäfer M, Scholz J and Greiner M, 2006 Phys. Rev. Lett. 96108701

[14] Moreno Y, Gómez J B and Pacheco A F, 2002 Europhys. Lett. 58630

[15] Kim B J, 2004 Europhys. Lett. 66819

[16] Kim D-H, Kim B J and Jeong H, 2005 Phys. Rev. Lett. 94025501

[17] Barrat A, Barthémy M, Pastor-Satorras R and Vespignani A, 2004 Proc. Natl. Acad. Sci. U.S.A. 1013747

[18] Asta L D, Barrat Alain, Barthélemy M and Vespignani A, 2006 J. Stat. Mech. 04 P04006

[19] Barrat A, Barthélemy and Vespignani A, 2005 J. Stat. Mech. 05 P05003

[20] Karsai M, Juhász R and lglói, 2006 Phys. Rev. E 73036116

[21] Wang W-X, Wang B-H, Yin C-Y, Xie Y-B and Zhou T, 2006 Phys. Rev. E 73026111

[22] Yan G, Zhou T, HuB, Fu Z-Q and Wang B-H, 2006 Phys. Rev. E 73046108

[23] Korniss G, 2007 Phys. Rev. E 75051121

[24] Wang W-X and Chen G, 2008 Phys. Rev. E 77026101

[25] Goh K-I, Kahng B and Kim D, 2001 Phys. Rev. Lett. 87278701

[26] Park K, Lai Y C and Ye N, 2004 Phys. Rev. E 70026109

[27] Holme P, Kim B J, Yoon C N and Han S K, 2002 Phys. Rev. E 65056109

[28] Rosvall M, Trusina A, Minnhagen P and Sneppen K, 2005 Phys. Rev. Lett. 94, 028701 